\title{
Supporting the scholar role in intensive care nursing
}

\author{
M. Melles*, A. Freudenthal, H. de Ridder \\ Department of Industrial Design, Delft University of Technology, Landbergstraat 15, 2628 CE Delft, The \\ Netherlands
}

\begin{abstract}
This study investigates how future informatics applications can support and challenge intensive care nurses (ICU nurses) to grow and learn continuously. To this end a research-and-design tool is introduced which is based on a model of the nursing process that starts from the idea that a nurse fulfills three different roles: the role of practitioner (using information immediately to base actions upon), the role of scholar (using information later on to learn from) and the role of human (coping with stress and dealing with emotions). In this paper the focus is on the scholar role. Twenty-eight intensive care staff members from six different hospitals were asked to recount an imposing experience from the perspective of each role. Regarding the scholar role, the participants mentioned 77 learning strategies they adopt for individual as well as organizational learning. Individual learning concerned reflection on former patient cases, reflection on current patient cases to anticipate a change in the patient's condition and reflection on personal behavior and decisions. Organizational learning concerned reflection on former patient cases. Examples of specific strategies were formal team evaluations focused on procedure and understanding the perspective of team members, being present at autopsies, and giving feedback on the nursing skills of colleagues. Based on these strategies design implications are defined for future nursing informatics applications, which will be presented.
\end{abstract}

Keywords: intensive care nursing, learning, design, nursing informatics application

\section{Introduction}

In order to provide high-quality care, hospitals require appropriate numbers of well-trained and motivated nurses. An urging international problem, however, is the increasing shortage of nursing staff. The Institute of Medicine [6] as well as the International Council of Nurses [1] indicated poor working environments as one of the main reasons for this shortage. Research into so-called positive practice environments, working environments that are able to support excellence and attract and retain nurses, indicates that nurses are more likely to be attracted to, and perform better in, hospitals in which they can advance professionally $[1,2]$. This study investigates how future informatics applications can support and challenge intensive care nurses (ICU nurses) to grow and learn continuously, in a way that

\footnotetext{
* Corresponding author. E-mail: m.melles@tudelft.nl
}

fits their natural working methods. Based on insights into the actual learning strategies of intensive care nurses design implications are defined for future nursing informatics applications. To this end a research-and-design tool is introduced [3]. This tool aims to support product developers in (1) collecting useful data by facilitating an effective discussion with intensive care staff about the work process of intensive care nurses and (2) to arrive at design implications on work support from the collected data. The tool is based on a model of the nursing process that starts from the idea that a nurse fulfills three different roles. A distinction is made between the nurse's role of practitioner (using information immediately to base actions upon), the nurse's role of scholar (using information later on to learn from) and the nurse's role of human (coping with stress and 
dealing with emotions). In this paper the focus is on this scholar role.

\section{Method}

\subsection{Participants}

Twenty-eight ICU staff members from six Dutch hospitals took part in this study, including eighteen ICU nurses and ten ICU staff members with a management function (i.e., five ICU head nurses and five staff advisors on medical equipment). These two participant groups were chosen because they were expected to have differing views on the needs of an ICU ward. The ICU nurses, with their accumulated, daily hands-on experience were expected to give input mainly about actual working methods and needs on the ward and at the patient's bedside. The management group was expected to provide opinions on the functioning and needs of the ICU ward as a whole and of the ICU ward as part of the larger hospital context. The work experience of the ICU nurses ranged from none (one person had just started working as an ICU nurse) to 25 years, with a mean of 11 years. Six nurses were male and twelve nurses were female. The management experience ranged from 4 to 17 years, with a mean of 10 years. All of the managers had previous nursing experience, ranging from 8 to 35 years. Six managers were male, four were female. Three of the six participating hospitals were teaching hospitals (twelve participating nurses, eight participating managers); the other three were regional hospitals (six participating nurses, two participating managers).

\subsection{Procedure}

The interview began with an explanation of the goal of the interview, i.e. investigating how ICU nurses actually work, learn and behave in an ICU working environment in order to come up with design goals and design directions for future nursing informatics applications. It was explained that the interview would be structured by means of a framework developed specifically for the context of ICU nursing and based on previous observations at ICUs and interviews with ICU staff. The three roles, practitioner, scholar and human, were described using the framework visualization shown in Figure 1. It was made clear to the participants that whenever something happens in the ICU the various roles will have an impact on how the situation is experienced or perceived, on the actions or strategies that are undertaken and on the information that is used or needed. The interviewer explained these roles are used as a means for product developers to observe and interpret the activities of ICU nursing with the aim of improving the design of new systems.

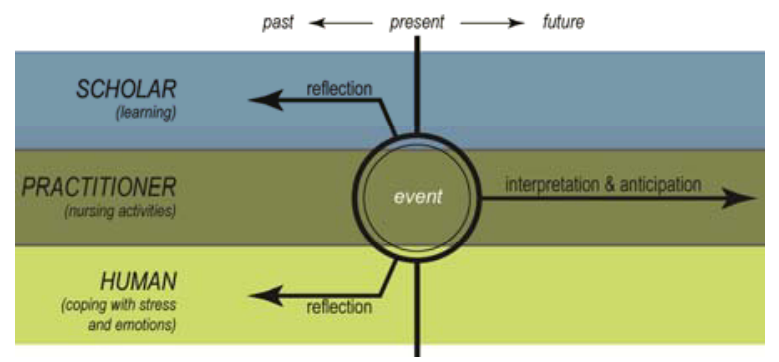

Figure 1 Overview of the three different roles that affect a nurse's perspective of a situation. Used during interviews to explain the roles concept to the participants (printed on A4, originally in Dutch).

Subsequently, the participants were asked whether they recognized the roles and agreed with this division. All acknowledged the existence of these roles. Then they were asked to recall a recent event on the ward in which all three roles were involved. This event could be anything; the only restrictions were that the participants had experienced it personally (meaning that it should not be an event experienced by a colleague or a description based on common knowledge) and, of course, that the event could be looked at from the perspective of each of the three different roles. First, the participants were asked to outline the situation in brief. Then they were asked to describe the different strategies adopted in each of the three roles, starting with the practitioner role, followed by the scholar role and concluding with the human role. If participants began to describe strategies in more general terms, they were encouraged to cast their mind back to the chosen situation and to describe only what was done in that specific situation.

The participants were asked to provide an extensive account of their work and their working methods. They were encouraged to think beyond the information read from equipment and to include information that was perceived directly from other sources, such as the patient, their colleagues, physicians, and the patient's relatives. In addition, the participants were encouraged to share ideas they had for future ICU systems. Sometimes the participant suggested product ideas spontaneously. Some other times the industrial design engineer 
conducting the interview came up with technical solutions to the problems mentioned right away and asked the participant's opinion of them during the interview. In this way a form of participatory design was adopted during the interview.

Nurses and managerial staff were asked the same questions. When the managerial staff did not have recent experience in patient care, they were asked to discuss events in more general terms. The interviewer used forms to note the event mentioned plus the corresponding strategies in each role to lead and focus the discussion. All interviews were audio recorded with the permission of the participants. The interview, including discussions on the practitioner and human role, lasted about 45 minutes. This paper presents the results concerning the scholar role. The results of the whole interview are presented in [3].

\subsection{Analysis}

Transcripts of all interviews were made. First, all of the design-relevant data were collected from each transcript, including (1a) the situation the participant described (e.g., a resuscitation setting), (1b) the specific problem $(s)^{2}$ the participant experienced in that situation (e.g., not clear which protocol to follow); (2) the strategies the participants mentioned adopting in order to deal with the situation for (a) the practitioner role (e.g., following the orders of the physician), (b) the scholar role (e.g., attending the formal team evaluation) and (c) the human role (e.g., having an informal discussion with colleagues); and (3) the ideas suggested by the participants during the interview and ideas suggested by the researcher and affirmed by the participant.

Next, all user data collected from the individual transcripts were combined in order to identify possible design goals and design directions. Design goals were defined as situations desirable for the future which can be achieved by means of new products or systems [7] and were based on the specific problems, or situational discontent experienced by the participants. Design directions were defined as possible solutions or indicators of solutions to meet the design goal. All problems mentioned by the participants were first grouped according to their common properties, such as uncertainty about the cause of a change in the

\footnotetext{
${ }^{2}$ Note that problems might also include difficult tasks.
}

patient's condition or poor team dynamics. The resulting problem groups were then grouped by situation and event. The resulting overview provided a first impression of potential design goals for ICU nursing support.

In addition, all strategies ${ }^{3}$ mentioned for each role were clustered by user goal. User goals indicate possible design goals for ICU nursing support. Furthermore, the strategies that nurses and managers adopt to achieve their goals indicate possible design directions. These strategies can be translated into design directions in three ways: (1) actions that can potentially be supported or enhanced by information technology: (2) an inventive (analogue) working method that inspires the product developer in defining a (digital) product function, (3) a current digital way of working that has the potential to be used for other purposes. Finally, the ideas suggested by the participants during the interview or suggested by the researcher and confirmed by the participants were labelled according to the design goal(s) to which they relate. Note that problems, strategies or idea suggestions that were mentioned only once or twice are also included in the results, because each event and approach mentioned by the participants can provide important leads for design [cf. 5, 9].

\section{Results}

\subsection{Events, situations and problems}

All participants shared one or more experiences. The nurses usually talked about one experience indepth, whereas most ICU managers discussed various experiences on a more general level. Because we asked the participants to describe only events that address all three roles, including the human role, most participants discussed rather stressful or emotional events that had remained in their memory.

The participants discussed 43 problems in total, 22 of which were brought up by the nurses and 21 by the managers. We identified sixteen different problems, relating to eight different situations. The situations in

\footnotetext{
${ }^{3}$ In this paper we use the term "strategies" instead of the term "tasks". Tasks are understood to be the official tasks of nurses, as stated in their contract, whereas we are interested in the strategies nurses actually adopt to achieve their goals.
} 
turn were clustered into four events ${ }^{4}$ : deterioration in the patient's condition, adverse event or near incident, change in the nursing or medical plan and introduction of new equipment.

Most problems addressed by the nurses relate to the event deterioration in the patient's condition (12 problems out of 22). Uncertainty about the cause of a deterioration in the patient's condition was mentioned most often, followed by poor team dynamics under time-critical circumstances, and discord with patient and relatives or the responsible medical specialist about the medical plan. Most issues addressed by the managers relate to the events deterioration in the patient's condition and adverse event or near incident. Discord about the medical policy with patient and relatives is mentioned most often by this group (e.g., a 14-year old patient transferred to the nursing ward, while his parents want him to stay in the ICU). Problems that relate to learning were mentioned by the ICU managers only. The nurses did not mention any problems in relation to learning.

The participants mentioned a total of 308 strategies (including duplicates) with respect to the three roles; 131 strategies concerned the practitioner role $(43 \%)$, 77 strategies concerned the scholar role $(25 \%)$ and 100 strategies concerned the human role $(32 \%)$. The nurses described 237 strategies (77\% of all strategies, an average of 13 strategies per nurse) and the managers mentioned 71 strategies $(23 \%$, an average of 7 strategies per manager). The nurses mentioned considerably more strategies than the managers, which was expected, since they have more hands-on experience.

\subsection{Strategies in the scholar role}

With respect to the scholar role, the participants put forward 77 strategies: 54 strategies were suggested by the nurses (70\%) and 23 by the managers (30\%) (see Table 1). Taking into account the ratio between the number of nurses and managers who participated $(64 \%$ to $36 \%)$, the number of strategies per participant is about the same (an average of 3 strategies per nurse and 2.9 strategies per manager). The strategies related to the scholar role are clustered into 20 unique strategies, which in turn are grouped

\footnotetext{
${ }^{4}$ Events are "things" that happen in the ICU work environment, over which the nurse does not always have direct control [cf. 10]; events trigger specific tasks or actions by the nurse.
}

into four user goals (see Table 1): reflection on former patient cases for individual learning, reflection on current patient cases to anticipate changes in the patient's condition, extending one's general nursing knowledge (also contributing to individual learning) and reflection on former patient cases for organizational learning. Nearly two-thirds of the strategies refer to the first two categories. The main difference between the first and the second category is the objective for reflecting. The second category refers to cases involving patients who have not been discharged, so that, contrary to the first category, the main objective of reflection is not individual learning but improved anticipation of changes in the patient's condition. The fourth category, reflection on former cases for organizational learning, is based on strategies mentioned only by the managers. More than half of the strategies mentioned are about learning from or with colleagues: formal team evaluations (Table 1, strategies $\mathrm{s} 1$ and $\mathrm{s} 7$ ), informal discussions with fellow nurses (s5 and s9) and indirect learning through providing feedback on the skills of others (s5), explaining your actions and strategies to trainees (s6) and answering questions (s17).

\subsection{Design implications for the scholar role}

Using the four user goals listed in Table 1 as a starting point, four design goals for digitally supporting the scholar role in ICU nursing were identified: support for reflection on former patient cases for individual learning, support for reflection on a current patient case for individual learning, support for reflection on personal behavior and decisions for individual learning, and support for reflection on former patient cases for organizational learning. These design goals and suggestions for implementation are described in the following paragraphs. The codes in parentheses in the text refer to the numbering of the strategies in Table 1.

Support for individual learning: reflection on former patient cases (S1)

Individual learning by reflecting on former patient cases is carried out by the nurse alone (strategies s2, s4 and s6) or in discussions with colleagues, either formal (s1, s7) or informal (s3, s5). Digital support for individual learning could therefore include individual solutions as well as team-based solutions. For both solutions, the support for individual learning should include a broad perspective on the patient 
case, including the process, all stakeholders involved and both the nursing and medical perspective on the patient case. Access to the perspective of others (fellow nurses or medical specialists) involved in the same case was mentioned as a means to evoke a deeper insight. In light of this, the use of evaluation forms can be extended. In some hospitals digital forms are used to evaluate reanimations. All of the medical staff involved are required to fill in this form. Currently, it is not always clear to the medical staff

Table 1

Overview of the strategies in the scholar role as mentioned by the ICU nurses (nrs) and managers (man).

\begin{tabular}{|c|c|c|c|}
\hline User goal & Strategy & \#nrs & $($ sub-) total \\
\hline \multirow{8}{*}{$\begin{array}{l}\text { S1. Individual learning: } \\
\text { Reflection on former } \\
\text { patient cases }\end{array}$} & s1. Formal team evaluation, focus on procedure & 5 & 3 \\
\hline & $\begin{array}{l}\text { s2. Personal reflection from memory, focus on } \\
\text { process }\end{array}$ & 4 & 0 \\
\hline & $\begin{array}{l}\text { s3. Attend patient autopsy and discuss the } \\
\text { outcome with medical specialists }\end{array}$ & 4 & 0 \\
\hline & s4. Fill out an evaluation form & 3 & 2 \\
\hline & s5. Informal discussion with fellow nurses & 3 & 0 \\
\hline & $\begin{array}{l}\text { s6. Run through the vital parameters measured by } \\
\text { equipment }\end{array}$ & 2 & 1 \\
\hline & $\begin{array}{l}\text { s7. Formal team evaluation, learning others' } \\
\text { perspectives }\end{array}$ & 1 & 0 \\
\hline & $\begin{array}{l}\text { s8. Use formal methods (e.g., STAR) for } \\
\text { reflection }\end{array}$ & 0 & 1 \\
\hline
\end{tabular}

\begin{tabular}{|c|c|c|c|c|}
\hline \multirow[b]{2}{*}{ S2. Individual learning: } & & 22 & 7 & $29(38 \%)$ \\
\hline & s9. Informal discussion with fellow nurses & 6 & 0 & \\
\hline \multirow{5}{*}{$\begin{array}{l}\text { Reflection on current } \\
\text { patient cases (in order to } \\
\text { anticipate changes in the } \\
\text { patient's condition) }\end{array}$} & s10. Ask advice of medical specialist & 3 & 2 & \\
\hline & s11. Look up general background information & 3 & 0 & \\
\hline & $\begin{array}{l}\text { s12. Check the vital parameters measured by } \\
\text { equipment }\end{array}$ & 2 & 1 & \\
\hline & s13. Check protocol & 2 & 0 & \\
\hline & $\begin{array}{l}\text { s14. Compare the current situation with the } \\
\text { memory of past experiences }\end{array}$ & 1 & 0 & \\
\hline
\end{tabular}

S3. Individual learning: Reflection on personal behavior and decisions

$\begin{array}{lrrr}\text { s15. Give feedback on the nursing skills of } & 7 & 0 \\ \text { colleagues (trainees) } & & \\ \text { s16. Explain one's own nursing skills to others } & 4 & 3 \\ \text { s17. Answer questions related to nursing } & 3 & 0 \\ \text { s18. Give feedback on the medical skills of } & 1 & 1\end{array}$
residents

\begin{tabular}{|c|c|c|c|c|}
\hline & & 15 & 4 & $19(25 \%)$ \\
\hline S4. Organizational & s19. Adopt a "helicopter view" & 0 & 8 & \\
\hline learning: Reflection on & s20. Give feedback to the ICU departments & 0 & 1 & \\
\hline & & 0 & 9 & $9(11 \%)$ \\
\hline total & & 54 & 23 & $77(100 \%)$ \\
\hline
\end{tabular}

what is done with these forms, as they do not receive feedback about what they filled in. However, these forms could be linked so that each nurse learns how the reanimation was experienced by other team members. In addition to its educational value, insight into the way colleagues experienced the same event contributes to an understanding of the behavior of team members. This contributes to better team 
dynamics in the future. Individual learning by means of a team evaluation should support individual reflection and should therefore be organized according to the same structure as the individual support. Team evaluations should include a complete roster of procedures involved in an event. Current team evaluations often focus only on the technical procedure (s1), although problems often stem from poor non-technical procedures, such as poor team dynamics.

Ideas suggested by the participants or suggested by the researcher and confirmed by the participants:

- Viewing a situation again by means of a video or electronic $\log$.

- Coupling of measured data to the emotional experience of a reanimation. Evaluation of all stages of the process. (also relates to design goal S4).

- Re-enacting an event in the skills lab.

- Compiling a checklist to help to think of all the important details when evaluating an event (also relates to design goal S4).

- Supporting the team evaluation with the objectively measured data.

- Timeline representing the whole process, including protocols and the tasks of all stakeholders involved (also relates to the design goals S2 and S4).

- Including all team members in decisions; giving them more responsibility to the organization as a whole (also relates to design goal S4).

- Having nurses exchange experiences with the aim of improving existing protocols or defining new protocols (also relates to the design goals S3 and S4).

- Including contextual factors when evaluating an event, for example by applying the "prisma" $\operatorname{method}^{5}[8]$ (also relates to design goal S4).

\section{Support for individual learning: reflection on current patient cases (S2)}

Reflecting on a current patient case is mainly aimed at obtaining a better understanding of the patient's

\footnotetext{
${ }^{5}$ PRISMA stands for Prevention and Recovery Information System for Monitoring and Analysis. The main goal of this method is to recover the root causes of incidents (including technical, organisational and human aspects) and to build a database from which conclusions may be drawn to suggest preventive measures [8].
}

condition in order to anticipate changes in an early phase and act promptly. An indirect result of such an activity is extending one's individual knowledge. When the condition of the patient is complex and not quite clear, it is a strong incentive for nurses to look up information to help obtain a good understanding of the case. Digital support should encourage the nurse to check all possible sources of information (s10, s11, s12, s13), including general knowledge on the Internet, protocols, the medical plan (ideally including medical motivations), the nursing plan (including motivations as well), and the patient's medical and nursing history. A checklist or a structure could, for example, provide support for analyzing a case. Also, support for comparing the case at hand with other, past cases would be a valuable addition (s14). An important addition would be the ability to couple protocols or other procedures to the case, so that the protocols could be personalized to the case and predictions about how the patient's condition might evolve could be made, including about the effects of possible actions.

Ideas suggested by the participants or suggested by the researcher and confirmed by the participants:

- Integrating the clinical view of the nurse and the actual data from the equipment.

- Timeline representing the whole process, including protocols and the tasks of all stakeholders involved (also relates to the design goals S1 and S4).

- Equipment providing suggestions about what the consequences of a particular action might be.

- Provision of a structure that obliges the nurse to work from problem analysis to actions (problem analysis, goal, actions, evaluation), making intuitive decisions tangible and gaps in one's work process visible.

- Comparison of the general course of an illness with the actual measured values in order to alert the nurse to variations in values.

- Database with an overview of patients with the same condition and their reactions to certain medication, supporting nurses in their choice concerning dosage and type of medication.

Note that the two design directions sketched above are based on two different incentives to reflect on cases. The main incentive for nurses to reflect on former patient cases is that, if something went wrong, nurses can find out whether they could have prevented it. The main incentive to reflect on current cases is to anticipate changes and hence be able to act promptly when necessary. 
Support for individual learning: reflection on personal behavior and decisions (S3)

An important learning aspect in ICU nursing is onthe-job training. This approach facilitates learning in two directions. The trainee learns about ICU nursing by observing and hearing explanations of what the registered nurse does. Additionally, and relevant to our research purposes, making one's actions explicit by explaining them to others elicits a learning experience for the instructor as well. Reflection on personal decisions could also be triggered by equipment, for example by providing a structure or checklist that invites nurses to reflect on their own actions. Another possibility is for technology to serve as a medium for nurses to exchange experiences, for example by providing the possibility of posting questions and answers or by providing the possibility of viewing other patient cases, including the nursing and medical decisions involved.

To go a step further, equipment could play an active role in defining feedback. For example, an infusion pump that can determine whether the nurse operates it correctly by the way it is used, thereby drawing the nurse's attention to useful functions that she currently does not use, or to more efficient operation sequences. In [4] it was explored and prototyped that this is actually technically possible and its value was verified in user tests. Another example was given by one of the managers participating in this study. He mentioned that some nurses have the tendency to note only the patient values in the patient data management system (PDMS) that do not require them to take action. Such behavior could be deduced by the system and fed back to the nurses.

Ideas suggested by the participants or suggested by the researcher and confirmed by the participants:

- A personal agenda reminding the nurse when specific equipment training is needed again.

- Equipment giving individual feedback based on how the device is operated (in particular, functions that are not used often, see also [4]).

- Deducing human behavior from the PDMS and feeding this back to the nurse (for example, a tendency to note only the most favorable values, hence those not requiring action).

- Having nurses exchange experiences with the aim of improving existing protocols or defining new protocols (also relates to the design goals S1 and S4).
Support for organizational learning: reflection on former patient cases (S4)

Currently, organizational learning from reflection on former patient cases is carried out mainly by managers, who aim to learn from the events and, based on the insights they derive, to implement organizational changes that will improve patient care. In order to understand the bigger picture around an event, ICU managers need a "helicopter view" of a case (s19), revealing for instance the whole chain of events, all of the protocols involved, the equipment uses, and all organizational influences. Digital support could include some kind of a checklist or visual structure to make managers aware of the aspects that should be considered. The influence of equipment design, for example, should be considered. The results of this "helicopter view" should be fed back to the departments so that the nursing staff can see and understand changes, as well as to give their own feedback about changes (s20). In this way, the nurses will understand the changes; they will feel part of the organization and also contribute to organizational changes that lead to higher-quality patient care. In addition, design-related issues should be fed back to the manufacturers of equipment.

Ideas suggested by the participants or suggested by the researcher and confirmed by the participants:

- Coupling of measured data to the emotional experience of a reanimation. Evaluation of all stages of the process (also relates to design goal S1).

- Compiling a checklist to help to think of all the important details when evaluating an event (also relates to design goal $\mathrm{S} 1$ ).

- Including contextual factors when evaluating an event, for example by applying the "prisma" method [8, see footnote 3] (also relates to design goal S1).

- Timeline representing the whole process, including protocols and the tasks of all stakeholders involved (also relates to the design goals S1 and S2).

- Including all team members in decisions; giving them more responsibility to the organization as a whole (also relates to design goal S1).

- Having nurses exchange experiences with the aim of improving existing protocols or defining new protocols (also relates to the design goals $\mathrm{S} 1$ and S3). 


\section{Discussion and conclusions}

This study introduces a way of structuring interviews based on the three different roles an ICU nurse is expected to fulfill, i.e. practitioner, scholar and human. This approach led to a variety of design goals and design directions for the digital support of ICU nursing. Four design goals have been identified for the scholar role aiming to support and challenge ICU nurses to grow and learn continuously. A future learning system should probably include all four goals. In order to come up with good solutions, it might still be useful to start designing from the different angles, with a different focus, keeping the other goals in the back of one's mind to explore the various possibilities.

The high number and level of design relevance of the collected user data demonstrates that our approach provides useful information for the development of digital support for ICU nursing. Many studies on ICU working methods do not directly imply specific design implications (other than general statements like "software usability is important to adoption"). With the results of this study we hope to make a useful and inspiring contribution to the development of future ICU systems. Although the results of our study are to be used for the development of digital products, the method and the results gathered can also be used as input for other purposes, for example, organizational changes or for training purposes. The approach of role-based interviews could, for example, be used to interview all multidisciplinary team members that were involved in a time-critical or even adverse event. The division of activities among the roles of the members involved during the event could give insight on how to optimize current protocols and procedures. In addition, the role-based interview structure could be used to evaluate a scenario which is play-acted during a team training. This way, team members could learn from each other's perspective on matters.

The interview structure used in this study could contribute to making nurses more aware of the products and product functions that could be designed for in their daily work. Nurses do not naturally think in design terms. They are more accustomed to adapting their work strategies to the equipment. The proposed structure gives the participants handles for viewing their work and for ways of discussing that suits the language of product developers. In view of this, it might be important for medical participants to better understand how their stories contribute to product development. In this study all the participants were quite cooperative in sharing their experiences and easily able to discuss them within the format of the roles. However, some of them mentioned that they had no clue what use product developers would put their stories to. More attention should probably be paid to explaining how their stories contribute to product development. This might be done by explaining the relation between their input and the resulting design implications, or by providing the participants insights into the results of the interviews or the resulting product ideas, for example by sending the participants the results afterwards.

\section{References}

[1] A. Bauman, Positive practice environments: quality workplaces $=$ quality patient care, Geneva, International Council of Nurses, 2007.

[2] H.K.S. Laschinger, Effect of empowerment on professional practice environments, work satisfaction, and patient care quality. Further testing the nursing worklife model, Journal of Nursing Care Quality 4 (2008), 322-330.

[3] M.Melles, Role-based support for intensive care nursing, Ph.D. Dissertation, Delft University of Technology, 2011.

[4] M. Melles, J.G.M. de Jong, A. Freudenthal and C.A.H.M. Bouwman, Error reduction through personalized assistance in medical equipment, Proceedings of the $16^{\text {th }}$ World Congress on Ergonomics, IEA, 2006.

[5] J. Nielsen, Usability engineering, Boston, Academic Press, 1993.

[6] A. Page, Keeping patients safe: transforming the work environment of nurses, Institute of Medicine, Washington, DC, National Academy Press, 2004.

[7] N.F.M. Roozenburg and J. Eekels, Product design: fundamentals and methods, West Sussex: John Wiley \& Sons, 1995.

[8] T.W. van der Schaaf and M.M.P. Habraken, PRISMAMedical, Eindhoven University of Technology, 2005

[9] A.P.O.S. Vermeeren, What's the problem, Ph.D. Dissertation, Delft University of Technology, 2009.

[10] M. van Welie, Task-based user interface design, VU University Amsterdam, 2001. 